\title{
The gamma-ray source-count distribution as a function of energy
}

\section{Hannes-S. Zechlin*}

Istituto Nazionale di Fisica Nucleare, Sezione di Torino, via P. Giuria, 1, I-10125 Torino, Italy

E-mail: zechlinato.infn.it

\begin{abstract}
Photon counts statistics have been shown to provide a sensitive tool for characterizing gamma-ray source populations and the composition of the gamma-ray sky. In this contribution, we extend the use of the standard 1-point probability distribution function (1pPDF) to decompose the highlatitude gamma-ray flux observed with Fermi-LAT into emission from point sources, Galactic foreground, and diffuse isotropic background. To that aim, we perform an energy-binned analysis of the gamma-ray data between 1 and $171 \mathrm{GeV}$. We measure the source-count distribution $\mathrm{d} N / \mathrm{d} S$ in several energy bins, and we demonstrate that our results extend current measurements driven by point-source catalogs to so far undetected source contributions. The source-count distribution as a function of flux is found to be compatible with a broken power law. Upper limits on further possible breaks as well as the angular power of unresolved sources are derived. We discuss the composition of the gamma-ray sky and future prospects and capabilities of the 1pPDF method.
\end{abstract}

35th International Cosmic Ray Conference - ICRC2017

10-20 July, 2017

Bexco, Busan, Korea

${ }^{*}$ Speaker. 


\section{Introduction}

The extragalactic gamma-ray background (EGB) [1] is one of the main observables characterizing the high-energy gamma-ray sky. It is composed of individual sources, resolved in source catalogs such as the Fermi Large Area Telescope Third Source Catalog (3FGL) [2], and of a diffuse, unresolved component that is nearly isotropic. The diffuse background component is commonly known as the isotropic diffuse gamma-ray background (IGRB). The IGRB has been detected with the Fermi Large Area Telescope (Fermi-LAT) [3] with unprecedented precision [4], at Galactic latitudes $|b|>20^{\circ}$ and for energies between $100 \mathrm{MeV}$ and $820 \mathrm{GeV}$.

The majority of point sources resolved in the 3FGL catalog have been associated with blazars. Given the two different flavors of the blazar population, flat spectrum radio quasars (FSRQs) typically exhibit soft gamma-ray spectra with power-law indexes around $\sim 2.4$, while the observed spectra of BL Lacertae (BL Lacs) objects are characterized by harder power laws with an average index of $\sim 2$.1. In general, different source populations can be characterized by their intrinsic source-count distributions $\mathrm{d} N / \mathrm{d} S$, denoting the number of sources $N$ per solid angle element $\Delta \Omega$ with integral fluxes in the interval $(S, S+\mathrm{d} S)$. Data-driven phenomenological models of the total source-count distribution suggest that the IGRB is at least partly composed of unresolved point sources, too faint to be detected with current instruments.

As opposed to conventional point-source detection methods (i.e. catalogs), statistical analysis techniques are capable of decomposing gamma-ray sky maps into their constituents $[5,6,7,8,9]$. In Refs. [8] and [9], henceforth Z16a and Z16b, we have shown that the statistics of photon counts of a pixelized sky map, i.e. the 1-point probability distribution function (1pPDF), can be used to measure the $\mathrm{d} N / \mathrm{d} S$ distribution of sources with high precision, and to decompose the gamma-ray sky. The $1 \mathrm{pPDF}$ method has allowed us to measure the $\mathrm{d} N / \mathrm{d} S$ in the regime of so far unresolved faint point sources, down to fluxes a factor of $\sim 10$ below nominal catalog detection thresholds. The analysis employed 6-year Fermi-LAT data for high Galactic latitudes, in five energy bands between 1.04 and $171 \mathrm{GeV}$. This contribution gives a brief overview of the results obtained in Z16b.

\section{1pPDF Method and Fermi-LAT Data}

We considered the total gamma-ray sky to be composed of three basic constituents, based on state of the art observations: (i) an isotropic population of gamma-ray point sources, described by a $\mathrm{d} N / \mathrm{d} S$ distribution, (ii) a diffuse Galactic foreground (GF) component, and (iii) a diffuse component including all contributions indistinguishable from diffuse isotropic emission. The $\mathrm{d} N / \mathrm{d} S$ distribution was parameterized with a multiply broken power law (MBPL), including $N_{\mathrm{b}}$ break positions and thus $N_{\mathrm{b}}+1$ power-law components connecting the breaks. The GF emission was incorporated with spatial and spectral templates. The normalization of the GF template, $A_{\text {gal }}$, was kept free to vary. The spectrum of the diffuse background emission was assumed to follow a power law with photon index $\Gamma=2.3$; its integral flux, $F_{\text {iso }}$, was implemented as a free fit parameter.

The $1 \mathrm{pPDF}$ was computed by utilizing the approach of probability generating functions as developed in Z16a. The likelihood of the data is represented by a product over the probabilities $P$ of measuring the number $k_{p}$ of photons in each individual pixel $p$. The total likelihood of the 
region of interest, covered with $N_{\text {pix }}$ pixels, for a given parameter vector $\Theta$ thus reads

$$
\mathscr{L}(\Theta)=\prod_{p=1}^{N_{\mathrm{pix}}} P\left(k_{p}\right) .
$$

Therefore, the likelihood takes into account the spatial morphology of the GF component. Parameter values were estimated from the profile likelihood (frequentist approach) generated for each fit parameter from the overall posterior distribution, as computed from Markov Chain Monte Carlo (MCMC) sampling of $\mathscr{L}(\Theta)$.

We used Fermi-LAT P7REP_CLEAN data in five energy bands for high Galactic latitudes $|b| \geq 30^{\circ}: 1.04-1.99,1.99-5.0,5.0-10.4,10.4-50.0$, and $50-171 \mathrm{GeV}$. Note that the last energy band was evaluated for Galactic latitudes $|b| \geq 10^{\circ}$ in order to provide sufficient statistics. Event selection and data processing are detailed in $\mathrm{Z16b}$.

\section{Results}

\subsection{Source-count Distribution}

In order to fit our model of the gamma-ray sky to the data, we employed the 1pPDF method developed in Z16a. The $\mathrm{d} N / \mathrm{d} S$ parameterization was fit by consecutively increasing the number of free breaks $N_{\mathrm{b}}$, singling out the simplest parameterization required to fit the data properly. The results are shown in Fig. 1. We found that the data were described sufficiently well by broken power-law $\mathrm{d} N / \mathrm{d} S$ distributions, with a break at comparably high fluxes. The power-law indices below the first break are compatible with values between 1.95 and 2.0. The highest energy band is compatible with a simple power-law with an index of $2.2_{-0.3}^{+0.7}$. The best-fit $\mathrm{d} N / \mathrm{d} S$ parameterizations are depicted by the solid blue lines in Fig. 1, which are shown only above the estimated sensitivity of the analysis. The corresponding uncertainty bands (68\% confidence level) are marked with blue shaded regions. The bands were derived allowing more degrees of freedom (i.e. a higher number of free breaks), yielding robust and realistic estimates. As demonstrated in the figure, the $\mathrm{d} N / \mathrm{d} S$ measurements are in good agreement with the source counts derived from standard source catalogs (see Z16a,b for details). The 1pPDF method extends the source-population sensitivity by almost one order of magnitude below the nominal catalog detection thresholds ${ }^{1}$.

Upper limits on a second (first, for the highest energy band) intrinsic break of $\mathrm{d} N / \mathrm{d} S$ are depicted by the blue arrows in Fig. 1 .

\subsection{Anisotropies}

A complementary measure of unresolved point-source contributions to the IGRB is provided by anisotropy measurements (e.g., [10]). The $\mathrm{d} N / \mathrm{d} S$ distribution is related to the autocorrelation angular power spectrum $C_{\mathrm{P}}$ by

$$
C_{\mathrm{P}}\left(S_{\mathrm{th}}\right)=\int_{0}^{S_{\mathrm{th}}} S^{2} \frac{\mathrm{d} N}{\mathrm{~d} S} \mathrm{~d} S,
$$

where $S_{\text {th }}$ denotes the flux threshold allowing individual source detection. Figure 2 compares the autocorrelation derived from our $\mathrm{d} N / \mathrm{d} S$ measurements with the complementary anisotropy analysis of Ref. [10]. The two analyses agree within uncertainties.

\footnotetext{
${ }^{1}$ Below the nominal detection threshold the catalog suffers incompleteness.
} 

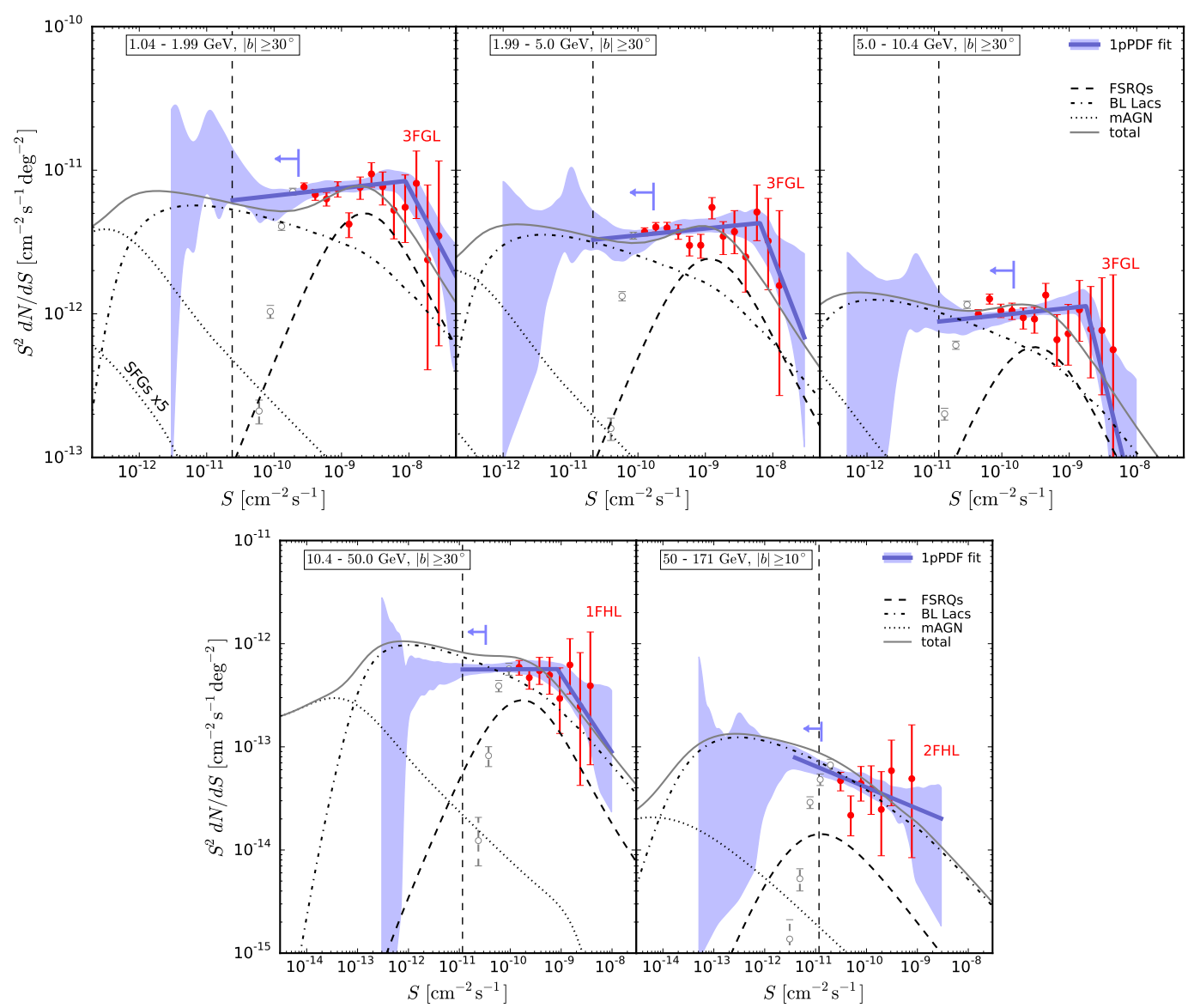

Figure 1: Differential source-count distributions $\mathrm{d} N / \mathrm{d} S$ obtained with the $1 \mathrm{pPDF}$ method. The best fits and uncertainties at $68 \%$ confidence level (CL) are depicted by the solid blue lines and the blue-shaded bands. The $\mathrm{d} N / \mathrm{d} S$ distributions derived from cataloged sources are shown by the red circles. The open gray circles depict $\mathrm{d} N / \mathrm{d} S$ points from sources below the nominal detection threshold; thus, they cannot be used for comparison. The blue arrows denote upper limits $(95 \%$ CL) on a first or second break, respectively. The vertical dashed lines indicate the estimated sensitivity of the analysis. The dashed, dot-dashed, and dotted lines depict model predictions for FSRQs, BL Lacs, and misaligned active galactic nuclei; see Z16b for details.

\subsection{Composition of the Gamma-ray Sky}

The decomposition of the gamma-ray sky is provided by the integral fluxes from the three considered components, i.e. the point-source flux $F_{\mathrm{ps}}$, the GF flux $F_{\mathrm{gal}}$, and the flux from the

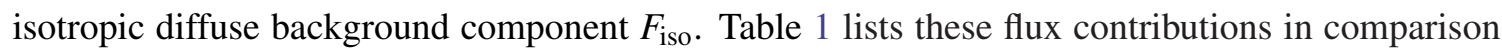
with the total flux from the high-latitude gamma-ray sky. The point-source contribution can also be expressed as fractional contribution to the extragalactic gamma-ray background (EGB), $F_{\mathrm{EGB}}$, as measured in Ref. [4]. The resulting contributions $F_{\mathrm{ps}} / F_{\mathrm{EGB}}$ in each energy band are $0.83_{-0.13}^{+0.07}$, $0.79_{-0.16}^{+0.04}, 0.66_{-0.07}^{+0.20}, 0.66_{-0.05}^{+0.28}$, and $0.81_{-0.19}^{+0.52}$, respectively. 


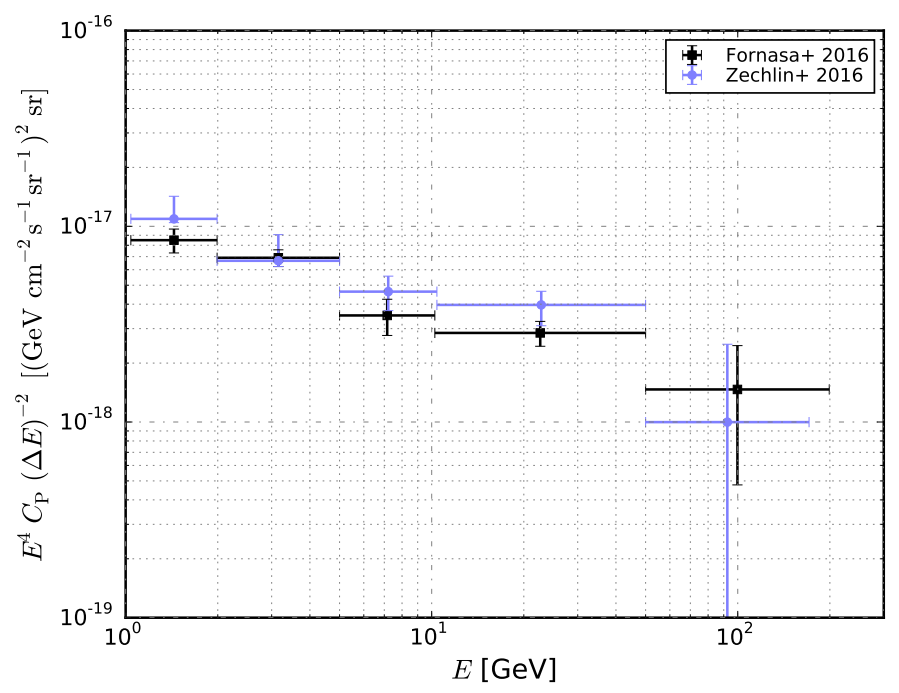

Figure 2: Autocorrelation $C_{\mathrm{P}}$ as function of the energy $E$. The $C_{\mathrm{P}}$ derived from the $1 \mathrm{pPDF}$ measurement of $\mathrm{d} N / \mathrm{d} S$ is denoted with blue circles, while the complementary anisotropy measurement of Ref. [10] is shown with black squares. (Remark: The analysis of Ref. [10] refers to the 3FGL catalog for source masking. We defined the anisotropy below the effective 3FGL detection threshold as $C_{\mathrm{P}}\left(S_{\mathrm{th}}^{3 \mathrm{FGL}}\right)$, see Eq. 3.1, such that $C_{\mathrm{P}}\left(S_{\mathrm{th}}^{3 \mathrm{FGL}}\right) \approx C_{\mathrm{P}}\left(S_{\mathrm{th}}^{<1}\right)-C_{\mathrm{P}}^{\mathrm{cat}}\left(S_{\mathrm{th}}^{<1}\right)$, where $C_{\mathrm{P}}^{\text {cat }}$ is the anisotropy contributed by cataloged (i.e. resolved) sources only, and $S_{\mathrm{th}}^{<1}$ approximates the nominal catalog detection threshold of the 3FGL.)

\begin{tabular}{lccccc}
\hline & $1.04-1.99 \mathrm{GeV}$ & $1.99-5.0 \mathrm{GeV}$ & $5.0-10.4 \mathrm{GeV}$ & $10.4-50.0 \mathrm{GeV}$ & $50-171 \mathrm{GeV}$ \\
\hline$q_{\mathrm{ps}}$ & $0.27_{-0.04}^{+0.02}$ & $0.27_{-0.06}^{+0.02}$ & $0.24_{-0.03}^{+0.08}$ & $0.27_{-0.03}^{+0.11}$ & $0.29_{-0.08}^{+0.19}$ \\
$q_{\text {gal }}$ & $0.714_{-0.005}^{+0.003}$ & $0.708_{-0.006}^{+0.005}$ & $0.598_{-0.01}^{+0.007}$ & $0.494_{-0.013}^{+0.008}$ & $0.49_{-0.02}^{+0.01}$ \\
$q_{\text {iso }}$ & $0.02_{-0.01}^{+0.04}$ & $0.012_{-0.001}^{+0.061}$ & $0.16_{-0.07}^{+0.03}$ & $0.23_{-0.15}^{+0.04}$ & $0.22_{-0.17}^{+0.10}$ \\
$F_{\text {tot }}$ & $9.17(1) \times 10^{-7}$ & $4.573(9) \times 10^{-7}$ & $1.103(3) \times 10^{-7}$ & $5.27(2) \times 10^{-8}$ & $5.67(6) \times 10^{-9}$ \\
\hline
\end{tabular}

Table 1: Composition of the gamma-ray sky. The quantities $q_{\mathrm{ps}}, q_{\mathrm{gal}}$, and $q_{\text {iso }}$ are the ratios of the integral flux components $\left(F_{\mathrm{ps}}, F_{\mathrm{gal}}, F_{\text {iso }}\right)$ and the total map flux $F_{\text {tot }}$. Parentheses denote symmetric errors on the preceding digit. The flux $F_{\text {tot }}$ is given in units of $\mathrm{cm}^{-2} \mathrm{~s}^{-1} \mathrm{sr}^{-1}$.

\section{Acknowledgments}

HSZ gratefully acknowledges the Istituto Nazionale di Fisica Nucleare (INFN) for a postdoctoral fellowship in theoretical physics on "Astroparticle, Dark Matter and Neutrino Physics", awarded under the INFN Fellowship Programme 2015.

\section{References}

[1] Fornasa, M., \& Sánchez-Conde, M. A. 2015, Phys. Rep., 598, 1

[2] Acero, F., Ackermann, M., Ajello, M., et al. 2015, ApJS, 218, 23

[3] Ackermann, M., Ajello, M., Albert, A., et al. 2012, ApJS, 203, 4

[4] Ackermann, M., Ajello, M., Albert, A., et al. 2015, ApJ, 799, 86 
[5] Malyshev, D., \& Hogg, D. W. 2011, ApJ, 738, 181

[6] Selig, M., Vacca, V., Oppermann, N., \& Enßlin, T. A. 2015, A\&A, 581, A126

[7] Lee, S. K., Lisanti, M., Safdi, B. R., Slatyer, T. R., \& Xue, W. 2016, Physical Review Letters, 116, 051103

[8] Zechlin, H.-S., Cuoco, A., Donato, F., Fornengo, N., \& Vittino, A. 2016, ApJS, 225, 18

[9] Zechlin, H.-S., Cuoco, A., Donato, F., Fornengo, N., \& Regis, M. 2016, ApJ, 826, L31

[10] Fornasa, M., Cuoco, A., Zavala, J., et al. 2016, Phys. Rev. D, 94, 123005 